\title{
Band Structure and Quantum Conductance of Metallic Carbon Nanotube Superlattices
}

\author{
W. JASKÓLSKI ${ }^{a}$, A. StAChÓW ${ }^{a}$ AND L. ChICO ${ }^{b}$ \\ ${ }^{a}$ Instytut Fizyki UMK, Grudziądzka 5, 87-100 Toruń, Poland \\ ${ }^{b}$ Facultad de Ciencias del Medio Ambiente \\ Universidad de Castilla-La Mancha, 54071 Toledo, Spain
}

\begin{abstract}
The electronic structure and quantum conductance of rotationally invariant $(6,6) /(12,0)$ and rotationally non-invariant $(5,5) /(8,2)$ superlattices made of metallic carbon nanotubes are investigated. It is shown that, except in the limit of very large periods, the quantum conductance of such superlattices does not critically depend on their rotational invariance, although it does in case of quantum dots and single junctions made of these nanotubes.
\end{abstract}

PACS numbers: 73.22.-f, 73.63.-b

\section{Introduction}

Carbon nanotubes $(\mathrm{CN})$ are one of the most promising candidates for a new kind of nanoelectronics. Their potential applicability in building of carbon-based nanodevices has been demonstrated by the construction of rectifying diodes [1], field-effect transistors [2], or carbon nanotube conducting networks [3]. In some of these applications one has to connect two or more different carbon nanotubes. Such connections (junctions) are most naturally realized by introducing pentagon/heptagon defects at the interfaces between the nanotubes. In some cases the interface can preserve rotational symmetries of the joined tubes; we will call it rotationally invariant (RI) junction. Such junctions can reveal conductance gaps, even if the constituent tubes are metallic [4]. Recently, we have shown [5] that a rotationally invariant quantum dot (RIQD) made by sandwiching a short portion of one metallic CN between two other metallic nanotubes, namely $(12,0) / N(6,6) /(12,0)$ 
(where $N$ denotes the number of $\mathrm{CN}$ unit cells), presents a series of localized nonconducting states appearing in a wide conductance gap. In contrast, rotationally non-invariant (RNI) metallic quantum dots, like e.g., $(8,2) / N(5,5) /(8,2)$, show a conductance gap with a series of narrow but conducting resonances [5]. Furthermore, we have recently shown [6] that in metallic carbon nanotube superlattices (SLs) made of a periodic sequence of RIQD, some of the above-mentioned localized states yield delocalized minibands, giving rise to a non-zero conductance. All these results indicate that the transport features of nanosystems built of carbon nanotubes strongly depend on the junction symmetry and structural details of the constituent tubes.

In this paper we perform a study of the electronic structure and transport properties of two different superlattices built of metallic carbon nanotubes: rotationally invariant (RI) and rotationally non-invariant (RNI) superlattices. In particular, we study $N(6,6) / N(12,0)$ and $N(5,5) / N(8,2)$ superlattices. We calculate the miniband structures, densities of states, and quantum conductances of these systems. We show that, unlike the single junction or quantum dots, the conductance of carbon nanotube superlattices built of short fragments of nanotubes does not critically depend on their rotational invariance.

\section{Theory}

We work in a tight-binding model with one $\pi$-orbital per atom. The nearest-neighbour hopping parameter is fixed to $V_{p p \pi}=t=-2.66 \mathrm{eV}$ and secondneighbour interactions are neglected. The electronic structure and transport around the Fermi level, $E_{\mathrm{F}}$, are well described by the $\pi$-orbital model [7]. Curvature effects are not addressed, since they are negligible for the nanotubes considered here.

There are 56 atoms in the $(8,2)$ unit cell and 20 atoms in the $(5,5)$ unit cell. The unit cell of $(12,0)$ contains 48 atoms and the unit cell of $(6,6)$ contains 24 atoms. Usually, CN unit cells are defined as uniform strips perpendicular to the nanotube axis [8], no matter whether the $\mathrm{CN}$ is chiral or not. But, when choosing a unit cell for a chiral tube with the purpose of designing a junction between two different tubes, it is often necessary to redefine it in order to assure that the junction only contains pentagons, heptagons, and hexagons. Obviously, there are multiple possible choices for the unit cell, yielding in general a variety of junctions between two tubes. In fact, the resulting junction may have a different number of pentagon/heptagon defects or just a different arrangement of these. This is what happens to the $(8,2)$ unit cell in the $(8,2) /(5,5)$ junction, and in fact, the properties of the $N(8,2) / N(5,5)$ superlattice may slightly vary depending on the choice of $(8,2)$ unit cell. The junctions appearing in two kinds of superlattices investigated in this paper are presented in Fig. 1. In the case of $N(6,6) / N(12,0)$ SL, the junction consists of 6 pentagon/heptagon pairs, and thus the superlattice 


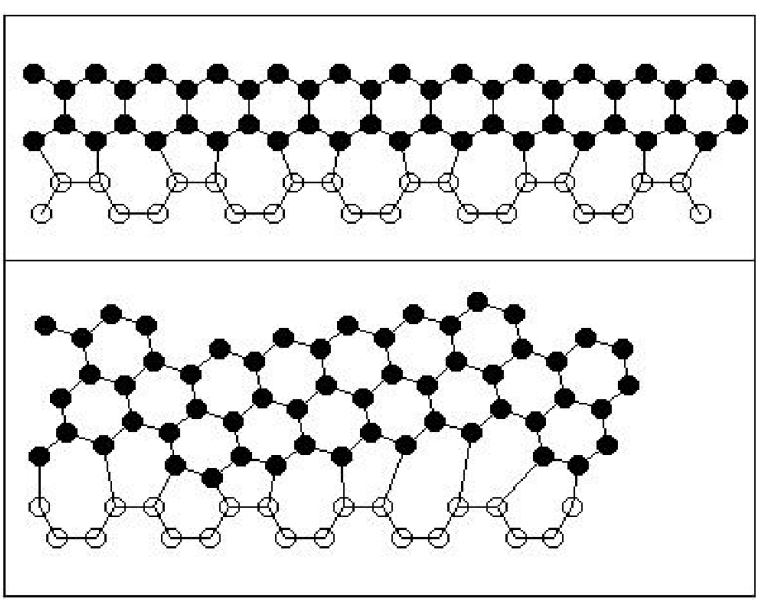

Fig. 1. Schematic (planar) view of the junctions in a rotationally invariant $(6,6) /(12,0)$ superlattice (top) and in a rotationally non-invariant $(5,5) /(8,2)$ superlattice (bottom). Open circles mark atoms of $(6,6)$ and $(5,5)$ unit cells.

is invariant under $2 \pi / 6$ rotations. In the case of the $N(8,2) / N(5,5)$ superlattice, the junction is built of a sequence of $(6,6,5,7,5,7,6,6,5,7)$ rings. The $(8,2)$ sections and the interface rings make this SL non-rotationally invariant.

The miniband structure for the investigated superlattices is calculated by diagonalization of the Hamiltonian matrix for the $k$ values in the first Brillouin zone. Next, the density of states (DOS) $\rho(E)$ is calculated as a histogram counting the number of $k$-states in a given interval $[E, E+\Delta E]$. The quantum conductance is calculated assuming that each non-degenerate miniband contributes with a conductance of $2 e^{2} / h$.

\section{Results}

The energy structure of any $N(n, m) / M\left(n^{\prime}, m^{\prime}\right)$ superlattice consists of a series of minibands. The minibands are formed in the following way: In any finite section containing $N$ unit cells of a given nanotube $(n, m)$, the bands $E_{n}(k)$ of this tube are quantized into a series of discrete localized states $E_{n}^{i}, i=1,2, \ldots$. These discrete states of the same energy $E_{n}^{i}$, localized in different $N(n, m)$ sections, form the minibands $E_{n}^{i}(k)$, since they can couple through the finite neighbouring sections $M\left(n^{\prime}, m^{\prime}\right)$. However, the energy structure of the finite adjacent section also consists of a series of discrete states $\tilde{E}_{n^{\prime}}^{j}$. Thus, the discrete energy levels $E_{n}^{i}$ can lie in the energy gaps of the neighbouring sections and therefore the coupling is through an energy gap (energy barrier) in such a case. If $N$ and $M$ are large, the coupling is weak and the minibands very narrow. However, if $N$ and $M$ are small, the coupling increases and the minibands get wider. It is also possible that two minibands $E_{n}^{i}$ and $\tilde{E}_{n^{\prime}}^{j}$, originating from discrete states that belong to different 
nanotubes, overlap. In this case, if (i) the superlattice is of RNI-type or (ii) it is of the RI-type and the bands, $E_{n}(k)$ and $\tilde{E}_{n^{\prime}}(k)$, have the same discrete angular momenta, the confinement of discrete states in the SL sections is due to wave vector mismatch, and the coupling has a resonant nature [5]. It can also happen that (iii) the superlattice is of the RI-type and the bands $E_{n}(k)$ and $\tilde{E}_{n^{\prime}}(k)$ have different discrete angular momenta; in this case, the coupling takes place through this symmetry barrier. Finally, some additional minibands can originate from the interface states [6].

The cases investigated in this paper cover the above-mentioned possibilities. Two RISL examples, namely $1(6,6) / 1(12,0)$ and $2(6,6) / 2(12,0)$, are shown in Fig. 2. In the first one, the $\mathrm{CN}$ sections are reduced to single unit cells and thus the minibands are extremely wide, while in the second case, the minibands are narrower, since the $\mathrm{CN}$ sections are twice longer. Closer inspection of the wave functions at $k=0$ allows us to recognize the origin of the minibands and the discrete rotation under which they are invariant. Minibands originating from quantized bands of $(6,6)$ and $(12,0)$ are denoted as $B$ and $X$ respectively; minibands originating from interface states are marked as $I$. The number $n$ added to these letters means invariance under $C_{n}=2 \pi / n$ rotation. As a reference, we give in the two bottom panels of Fig. 2 the band structure of the two constituent tubes, indicating the symmetry of the more relevant bands. This allows us to trace back the origin of the SL minibands. Thus, B6 indicates that the corresponding band is invariant under $C_{6}$; for a $(6,6)$ tube, this means completely symmetric

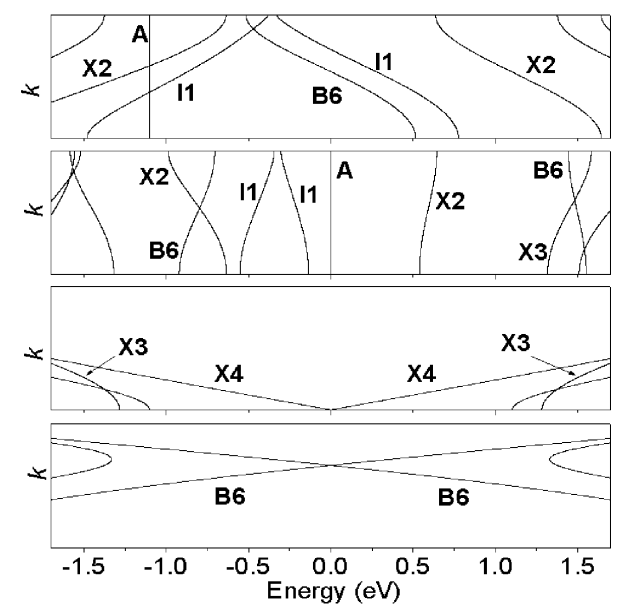

Fig. 2. From top to bottom: miniband structure of $1(6,6) / 1(12,0)$ SL; miniband structure of $2(6,6) / 2(12,0) \mathrm{SL}$; band structure of $(12,0) \mathrm{CN}$; band structure of $(6,6) \mathrm{CN}$. Only bands around $E_{\mathrm{F}}$ are shown. $B$ marks minibands originating from the $(6,6)$ tube, $X$ marks minibands originating from the $(12,0)$ tube, $I$ marks interface minibands, and $A$ marks dispersionless bands. The number $n$ added to the letters means invariance under $2 \pi / n$ rotation. 
wave functions. The interface states $I 1$ have no rotational symmetry, i.e., they are only invariant under rotations of $2 \pi$. Notice that, although the $(12,0)$ tube has $C_{4}$-invariant states, this symmetry is no longer present in the SL, so the minibands originating from the $X 4$ bands have their symmetry lowered to $C_{2}$, as can be seen in Fig. 2. As the discrete angular momenta of the $(12,0)$ and $(6,6)$ bands close to $E_{\mathrm{F}}$ are different, the miniband coupling takes place through a symmetry barrier. This also applies to the coupling of interface states.

Let us now consider the density of states of several rotationally invariant $N(6,6) / M(12,0)$ superlattices. We have recently [6] studied the properties of such superlattices for some particular values of $N$ and $M$. The miniband structure of any $N(n, n) / M(2 n, 0)$ SL always contains $2(N+1)$ dispersionless (DL) bands. Two of them have energies $\pm t$ and are doubly degenerate. Another $2 N$ DL bands are non-degenerate and their energies only depend on the lengths of the $N(n, n)$ section. A curiosity about DL bands is that the corresponding wave functions are exclusively localized in the $N(n, n)$ sections and pentagon/heptagon interfaces, although they originate from the dispersionless bands of the $(2 n, 0)$ nanotube [6]. In Fig. 2 they are marked as $A$.

Figure 3 shows a sequence of the DOS for several RISL, $N(6,6) / N(12,0)$ with $N=1,2,3,4$. The DOS is presented only in the range $E_{\mathrm{D}}=(-1.7,+1.7) \mathrm{eV}$ around the Fermi level, which is set equal to zero in the $\pi$-electron approximation. For the shortest SL, i.e., for $N=1$, one expects a strong quantization of the bands of $(12,0)$ and $(6,6)$ nanotubes and a large separation of the discrete energy levels. However, strong coupling through the single unit cell of the contiguous sections leads to wide and overlapping minibands (see Fig. 2) that cover all the

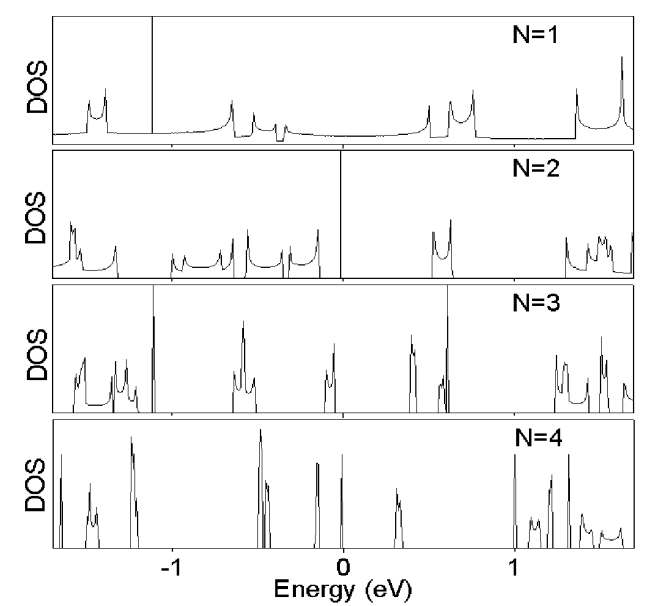

Fig. 3. Density of states for several $N(6,6) / N(12,0)$ SL; $N=1,2,3,4$ from top to bottom. 
investigated energy range. As a consequence, there is no energy gap, so that no conductance gap appears (see Fig. 4). When $N$ increases, the minibands get narrower and the energy gaps open up. It is interesting to note that when $N$ increases, the contribution of the miniband continua to the investigated energy range $E_{\mathrm{D}}$ decreases. In the case of very large $N, E_{\mathrm{D}}$ is densely covered by $\delta$-like minibands originating from the bands of the $(12,0)$ and $(6,6)$ tubes, which have different discrete angular momenta. It has a direct consequence on the quantum conductance, since for increasing $N$ the narrowing minibands do not practically yield any non-zero conductance. This effect is shown in Fig. 4.

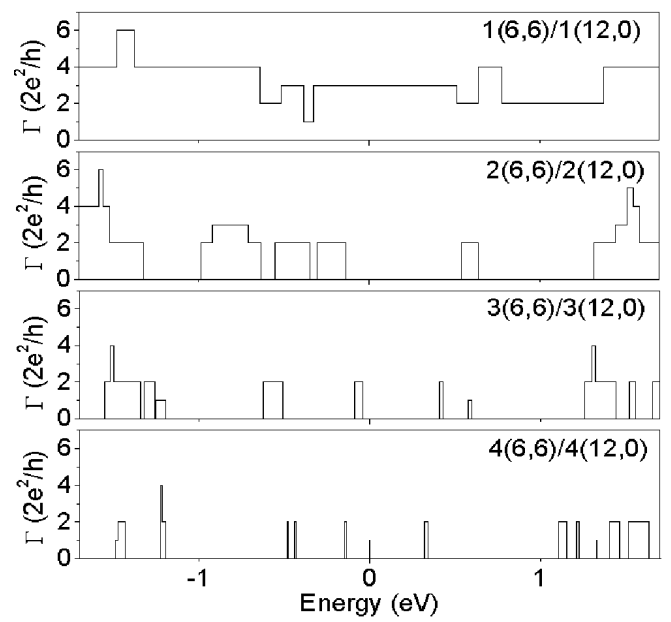

Fig. 4. Conductance of superlattices shown in Fig. 3.

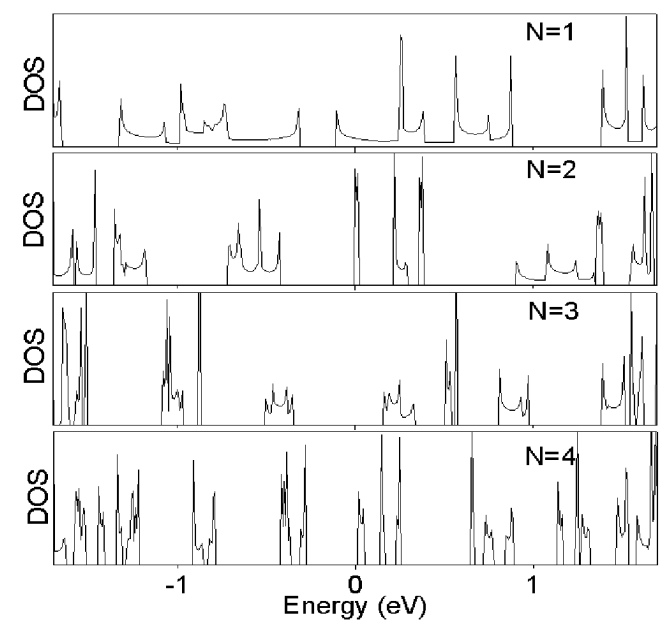

Fig. 5. Density of states for several $N(5,5) / N(8,2)$ SLs; $N=1,2,3,4$ from top to bottom. 
Let us now consider a RNISL made of two metallic nanotubes: $N(5,5) / N(8,2)$. In this case, the coupling of discretized bands through the neighbouring sections and subsequent formation of minibands is only due to energy barrier or wave vector mismatch. For small $N(N=2,3,4)$ there is no substantial difference between the DOS of this SL shown in Fig. 5 and that of the corresponding RISL (Fig. 3), except for the energy gaps, which already appear for $N=1$. In both cases the DOS has a structure of narrow minibands separated by minigaps. The conductance, shown in Fig. 6, is also similar in both cases. This is an intriguing observation, since significant differences in the quantum conductance of RI and RNI systems have been reported for both, the quantum dots and single junctions built of metallic nanotubes.

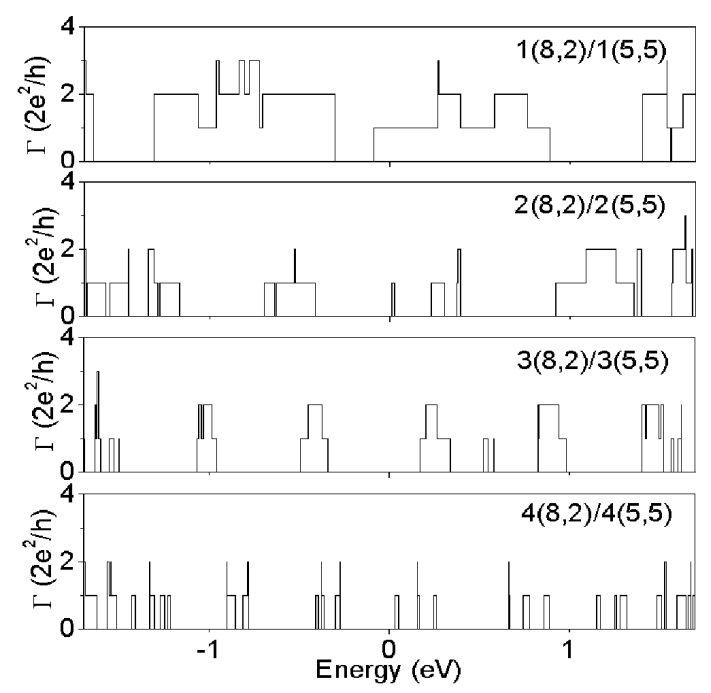

Fig. 6. Conductance of superlattices shown in Fig. 5.

However, when $N$ increases the investigated energy range becomes densely covered by minibands that originate from the bands of the $(8,2)$ and $(5,5)$ tubes, for which no symmetry restriction exists. In the limiting case of $N \rightarrow \infty$, all these conducting minibands form a conducting continuum.

\section{Conclusions}

In summary, we have investigated the electronic structure and quantum conductance of two types of superlattices built of metallic carbon nanotubes: $N(6,6) / N(12,0)$ and $N(5,5) / N(8,2)$. The main difference between these two superlattices is that the first one is rotationally invariant, while the second one is not. We have shown that conductance of superlattices built of metallic carbon nanotubes does not critically depend on their rotational invariance for short or intermediate periods. This behaviour significantly differs from that of single junc- 
tions and quantum dots built of such nanotubes, where a strong dependence of conductance on the rotational invariance was observed.

\section{Acknowledgments}

This work has been supported by the State Committee for Scientific Research grants 3T1104326 and PZB-MIN-008/P03/2003 (Poland) and Spanish grants MAT2002-04540-C05-03(DGES) and GR/MAT/0440/2004 (Comunidad Autónoma de Madrid).

\section{References}

[1] J.U. Lee, P.P. Gipp, C.M. Heller, Appl. Phys. Lett. 85, 145 (2004).

[2] S.J. Tans, A. Verschueren, C. Dekker, Nature 393, 49 (1998).

[3] M. Staderman, S.J. Papadakis, M.R. Falvo, J. Novak, E. Snow, Q. Fu, J. Liu, Y. Fridman, J.J. Boland, R. Superfine, S. Washburn, Phys. Rev. B 69, 201402 (2004).

[4] L. Chico, L.X. Benedict, S.G. Louie, M.L. Cohen, Phys. Rev. B 54, 2600 (1996).

[5] L. Chico, W. Jaskólski, Phys. Rev. B 69, 085406 (2004).

[6] W. Jaskólski, L. Chico, Phys. Rev. B 71, 155405 (2005).

[7] D. Orlikowski, H. Mehrez, J. Taylor, H. Guo, J. Wang, C. Roland, Phys. Rev. B 63, 155412 (2001).

[8] R. Saito, G. Dresselhaus, M.S. Dresselhaus, Physical Properties of Carbon Nanotubes, Imperial College Press, London 1998. 